Bull. Mater. Sci., Vol. 6, No. 3, July 1984, pp. 595-602. (C) Printed in India.

\title{
High voltage electron microscope irradiation and observations in metallic glasses
}

\author{
R V NANDEDKAR and A K TYAGI \\ Materials Science Laboratory, Reactor Research Centre, Kalpakkam 603 102, India
}

\begin{abstract}
High voltage electron microscope (HVEM) has been extensively used to produce radiation damage and to study the characteristics of defects so produced in crystalline solids. To understand the defect production in metallic glasses and to evaluate the influence of such defects on physical properties like crystallisation temperature etc., high voltage microscopy and subsequent in situ heating and observation has been extremely useful technique. This paper gives a qualitative overview of such work performed in metallic glasses. In particular results obtained on a nickel based metallic glass using a HVEM and an electron accelerator are presented. The advantages and limitations of HVEM irradiation are highlighted.
\end{abstract}

Keywords. Accelerator irradiation; metallic glass; defect characteristics; high voltage electron microscopy; crystallization temperature.

\section{Introduction}

The use of high voltage electron microscope (HVEM) as an electron irradiation facility to create displacement damage in crystalline samples is well known (Makin 1971). Electron irradiation will produce Frankel defects in metallic samples which will cluster into a complicated structure such as dislocation loops and voids which can simultaneously be observed when the irradiation is in progress. This technique has been applied very successfully to simulate fast reactor damage and get a quick estimate of the extent of damage that would occur in the fast reactor (Norris 1972a, b).

Metallic glasses have gained considerable interest both in basic and applied sciences. As far as 'defects' in amorphous systems in general and metallic glasses in particular are concerned, very little is so far known. A lot of experimental work backed with theoretical modelling has to be done before 'defects' in such systems could be understood. The study of production of defects, their recovery and influence on the mechanical, physical properties and crystallisation behaviour in metallic glasses can give an insight of the structure of amorphous materials. It is also suggested (Chang and $\mathrm{Li}$ 1977) that because of the nature of amorphous state, no more disorder can be introduced into metallic glasses by irradiation and if it be the case, these alloys are potential candidates for the nuclear energy applications. However radiation damage effects such as swelling (Chang and Li 1977) and blistering (Nandedkar and Tyagi 1981a) has been observed in the metallic glasses. The interesting area of research is the influence of these defects on the crystallisation behaviour. As already mentioned, HVEM offers a two-in-one, in the sense that one can produce and observe damage simultaneously. The effect of these defects on the crystallisation temperature $T_{c}$ can be found out by using the heating-stage and study the electron diffraction pattern as a function of temperature after irradiation. 
In this paper we give a qualitative survey of HVEM irradiation and accelerator irradiation work done to study defects and its influence on the crystallisation temperature of metallic glasses. The advantages and limitations are highlighted along with the results on a nickel based metallic glass $\left(\mathrm{Ni}_{45} \mathrm{Fe}_{5} \mathrm{Co}_{20} \mathrm{Cr}_{10} \mathrm{Mo}_{4} \mathrm{~B}_{16}\right)$ using a HVEM and an electron accelerator. These results are discussed by comparing with other experimental results.

\section{Survey of the earlier work}

Neutron irradiation (Lesueur 1970, 1975; Krammer et al 1979; Doi et al 1979) and charged particle (Richtin et al 1978; Schumacher et al 1980) irradiation experiments of metallic glasses so far have been able to establish the irradiation induced defects in metallic glasses. In these experiments techniques used were mainly wide-angle x-ray scattering, electrical resistivity, transmission electron microscopy etc. These techniques especially wide-angle scattering and resistivity have shown that irradiation produces 'vacancy type' of defects in metallic glasses. Another technique which is very sensitive to the presence of defects in the crystalline samples is positron annihilation. This technique includes positron lifetime and two-photon angular correlation measurements. This technique is very sensitive to very small vacancy concentration ranging from $10^{-4}$ to $10^{-7}$. Chen (1976) exploited this technique for electron irradiated (electrons from an accelerator) Pd-Ni-Si sample and the lifetime measurement did not show any evidence of vacancy like defects. Even two-photon angular correlation experiment(Chaung et al 1978) on electron irradiated Pd-Ni-Si showed no vacancy-like defects. Very recent experiments (Moser et al 1981) on positron lifetime measurements of low temperature electron irradiated metallic glasses show that lifetime increases after irradiation and comes back to original value after isochronal annealing. This experiment suggests that irradiation produces cavities of different sizes. These cavities are produced in the neighbourhood of the compressed regions of the metallic glasses. The recovery is explained on the basis of recombination of cavities with compressed regions. Electrical resistivity of low temperature $(4.6 \mathrm{~K})$ irradiated $\mathrm{Pd}_{80} \mathrm{Si}_{20}$ with electron of energy $3 \mathrm{MeV}$ is found to increase (Klaumunzer and Petri 1982; Klaumunzer et al 1981). The change in the resistivity is proportionate to radiationinduced defect concentration. Thermal recovery of $\mathrm{Pd}_{80} \mathrm{Si}_{20}$ is continuous from $10 \mathrm{~K}$ to $500 \mathrm{~K}$. These experimental results have been analysed by characterising the defects as excess free volume (vacancy-like defect) and negative free volume or compressed region (interstitial like defect). These results also indicate that the two types of defects have to be separated by a minimum distance of $\sim 10 \AA$ otherwise the stress field would overlap and give rise to spontaneous recombination.

The experiments above have so far shown that vacancy-type defects are produced. What influence will these 'defects' have on the crystallisation temperature $T_{c}$ ? Not much work has been done in the field of electron irradiation and its effect on $T_{c}$. However, people have effectively used HVEM in combination with heating-stage for $T_{c}$ determination. Kiritani et al $(1977,1978)$ studied the structure and crystallisation process of amorphous $\mathrm{Au}-\mathrm{Si}, \mathrm{Fe}-\mathrm{B}$ and $\mathrm{CoFeSiB}$ after electron irradiation in a HVEM. Only $\mathrm{Au}-\mathrm{Si}$ was prepared by vacuum deposition whereas other samples were prepared by melt quenching. They observed a lowering in the crystallisation temperature. In other words, the process of crystallisation is accelerated. This may be possible due to enhanced 
nucleation triggered off on account of radiation damage. The results are explained on the basis of variable latent vacancy mechanism of diffusion in amorphous materials (Kiritani and Yoshiie 1978). Doi et al (1976) have studied splat-cooled Fe-P-C and Fe-P in a HVEM with various energy levels from $100 \mathrm{keV}$ up to a dose of $1 \times 10^{23} \mathrm{e} / \mathrm{cm}^{2}$. They observed no appreciable change in the transmission electron diffraction pattern at room temperature. They observed that crystallisation is retarded or $T_{c}$ is enhanced. They explained the retardation on the basis that during splat quenching, clusters less than $10 \AA$ in size (atomic assemblies which are short range in order) are supposed to be present. These clusters act as nuclei for crystallisation. Irradiation destroys these nuclei and therefore there will be a low probability for forming the clusters or nuclei in the irradiated region and hence retardation in crystallisation. However the same authors have observed acceleration of crystallisation in FeBSi, NiBSi, NiBSiC, NiPB and NiP alloys and no effect in NiPC (Doi and Imura 1978, 1980). Metallic glasses 2826 $\left(\mathrm{Fe}_{40} \mathrm{Ni}_{40} \mathrm{P}_{14} \mathrm{~B}_{6}\right)$ and $2826 \mathrm{~A}\left(\mathrm{Fe}_{32} \mathrm{Ni}_{36} \mathrm{Cr}_{14} \mathrm{P}_{12} \mathrm{~B}_{6}\right)$ have been studied by Von Heimendahl (1981) after irradiation in HVEM which was operated at $1 \mathrm{MeV}$ and $1.1 \mathrm{MeV}$ to a dose of $4 \times 10^{23} \mathrm{e} / \mathrm{cm}^{2}$. It was observed that there is an acceleration in $T_{c}$ of $2826 \mathrm{~A}$ in the irradiated region. No actual $T_{c}$ measurement was done. In contrast, 2826, no effect of irradiation was visible. The explanation, which is rather qualitative, is put forward on the basis that $2826 \mathrm{~A}$ has low activation energy for nucleation of crystal $(350 \mathrm{~kJ} / \mathrm{mol})$ as against that of $2826(740 \mathrm{~kJ} / \mathrm{mol})$. The lower value of $2826 \mathrm{~A}$ explains the higher sensitivity to local rearrangements of atoms and therefore acceleration in $T_{c}$.

\section{Electron irradiation of a nickel based metallic glass}

HVEM offers many advantages to study samples over a conventional transmission electron microscope. Higher energy of electrons will give greater penetration $(\sim 1 \mu \mathrm{m})$ and therefore the information obtained is closer to a bulk sample. Due to higher beam energy one gets better practical resolution and a very good resolution in thinner samples. It also offers as mentioned earlier, electron irradiation facility to study radiation damage.

Although these advantages look rather attractive some limitations are there due to higher beam energy. Radiation damage poses some problems which are not desirable. It gives a greater danger to the operator from $x$-radiation from different parts of the microscope. It has poor response to the viewing screen, lower contrast, poor photographic emulsion and of course the high cost.

When used as irradiation facility to study and observe irradiation damage, it has limitations from the thickness. It is well known that the surfaces act as sink for the point defects produced during irradiation. In thin samples surfaces act as sinks and therefore it gives serious limitations when one wants to simulate neutron damage. Again electron irradiation will produce Frenkel pairs as against cascades in neutron and charged particle irradiation. One cannot irradiate in HVEM under controlled conditions on the sample such as temperature, stress. Therefore the only advantage it gives is to accumulate higher damage in shorter time due to high beam intensity. In an electron accelerator irradiation, one can have control over the specimen parameters such as temperature and stress. Thicker samples which are more representative of bulk can be used in an electron accelerator irradiation.

In studying the effect of irradiation on $T_{c}$ of metallic glasses in HVEMS, the only 
technique one can employ is the in situ heating in the hot-stage. The temperature of the sample is measured with a platinum-platinum rhodium thermo-couple which is attached to the holder and not to the sample. Depending upon the thermal contact of the specimen with the holder and the thermal conductivity of the sample, the temperature of the sample will be different from what is actually measured.

Judging all these limitations of HVEM irradiations we have irradiated a nickel-based metallic glass both in an electron accelerator to low doses and in a HVEM to a high dose. This metallic glass with composition $\mathrm{Ni}_{45} \mathrm{Fe}_{5} \mathrm{Co}_{20} \mathrm{Cr}_{10} \mathrm{Mo}_{4} \mathrm{~B}_{16}$, is in the form of a long ribbon about $40 \mu$ thick and $4 \mathrm{~mm}$ wide. Samples were irradiated to doses ranging from $1 \times 10^{14} \mathrm{e} / \mathrm{cm}^{2}$ to $4 \times 10^{16} \mathrm{e} / \mathrm{cm}^{2}$ with $3.5 \mathrm{MeV}$ electrons at room temperature using a water cooled target holder in a linear accelerator at Tata Institute of Fundamental Research, Bombay. For a high dose of $2 \times 10^{21} / \mathrm{cm}^{2}$, electron transparent samples were irradiated with $1 \mathrm{MeV}$ electrons in a JEM 125 HVEM of University of Antwerp, Belgium. The current density during HVEM irradiation was kept $1 \times 10^{21} \mathrm{e} / \mathrm{cm}^{2}$ min which roughly corresponds to a dose rate of $18 \mathrm{dpa} /$ hour. The rise in temperature due to beam heating was estimated to be $\sim 50 \mathrm{~K}$.

The crystallisation temperature $T_{c}$ is the temperature at which the process of crystallisation starts. This temperature $T_{c}$ was determined for both as-received and accelerator irradiated samples using resistometry, hot-stage electron microscopy and differential scanning calorimetry (Nandedkar and Tyagi 1981b; Nandedkar et al 1981). $T_{c}$ of HVEM irradiated sample was determined by heating the sample in situ in the hot stage of HVEM. Transmission electron microscopy samples for hot stage were prepared using $10 \% \mathrm{H}_{3} \mathrm{PO}_{4}$ and $90 \% \mathrm{H}_{2} \mathrm{SO}_{4}$ at $6 \mathrm{~V}$ and examined in hot-stage of Philips EM 400 operated at $100 \mathrm{kV}$. It was rather difficult to control the rate of heating but it is estimated to be about $15-20 \mathrm{~K} / \mathrm{min}$. HVEM samples were directly heated in situ after irradiation and the electron diffraction patterns were taken at different temperatures. The temperature at which the pattern changes from amorphous to crystalline is recognised as the crystallisation temperature. Figure 1 shows a typical hot-stage electron diffraction pattern of the accelerator irradiated sample. The crystallisation begins at $653 \pm 20 \mathrm{~K}$ in both as-received and accelerator irradiated samples. Figure 2 shows the typical micrograph and diffraction pattern of HVEM irradiated sample before and after the onset of crystallisation. The crystallisation was observed at a temperature $653 \pm 20 \mathrm{~K}$. Table 1 summarises the results on crystallisation temperature for asreceived, accelerator irradiated and HVEM irradiated metallic glass.

All the earlier studies on the effect of electron irradiation on crystallisation behaviour of metallic glasses were performed in HVEMS. Koster and Herald (1981) have pointed out that although in situ observation of crystallisation in hot-stage of a TEM will give

Table 1. Crystallisation temperature (in $\mathrm{K}$ ) of electron irradiated metallic glasses.

\begin{tabular}{lccc}
\hline & As-received & $\begin{array}{c}\text { Accelerator } \\
\text { irradiated }\end{array}$ & $\begin{array}{c}\text { HVEM } \\
\text { irradiated }\end{array}$ \\
\hline $\begin{array}{l}\text { Resistometric } \\
\begin{array}{l}\text { Hot-stage } \\
\text { electron } \\
\text { microscopy }\end{array}\end{array}$ & $390 \pm 15 \mathrm{~K}$ & $663 \pm 15 \mathrm{~K}$ & \\
D.S.C. & $673 \pm 5 \mathrm{~K}$ & $676 \pm 5 \mathrm{~K}$ & \\
\hline
\end{tabular}



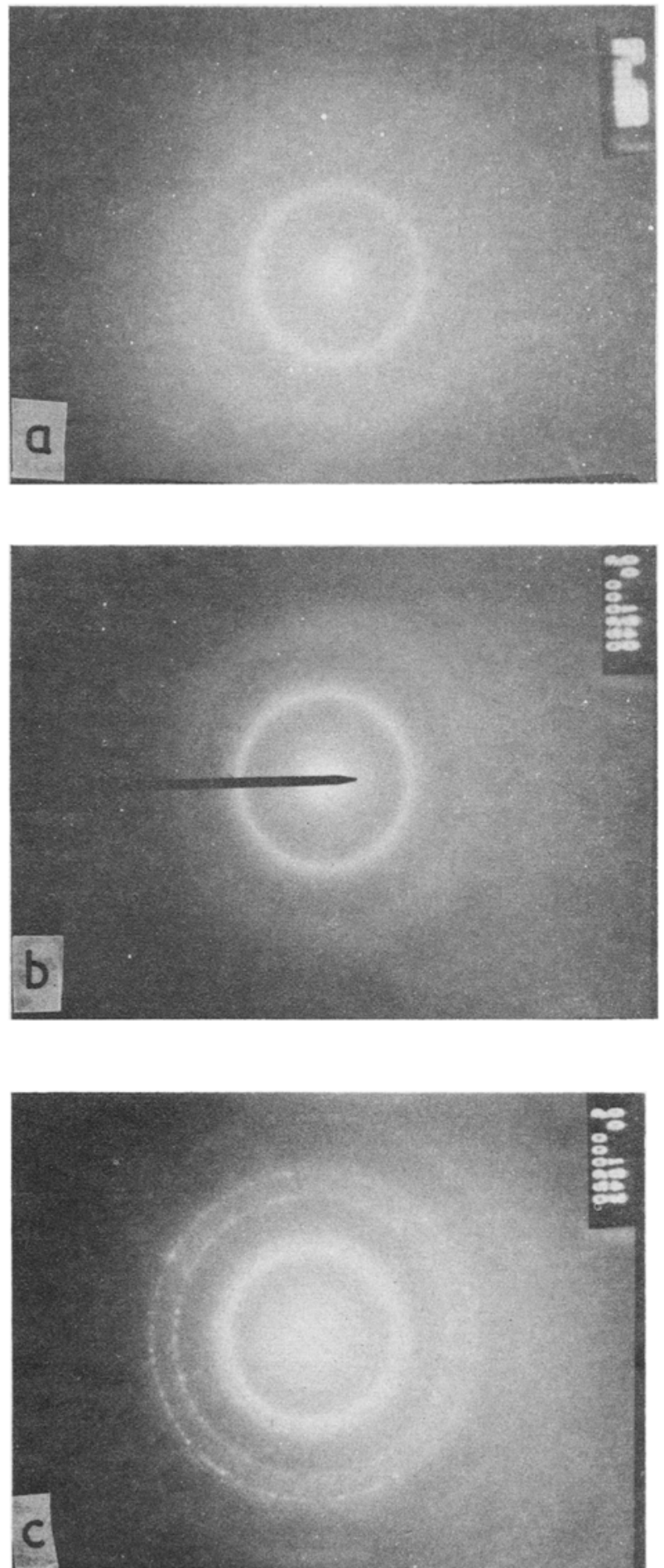

Figure 1. Electron diffraction pattern of electron irradiated (accelerator) metallic glass at a. room temperature b. $653 \mathrm{~K}$ and c. $703 \mathrm{~K}$. The crystallisation begins at $653 \mathrm{~K}$. 

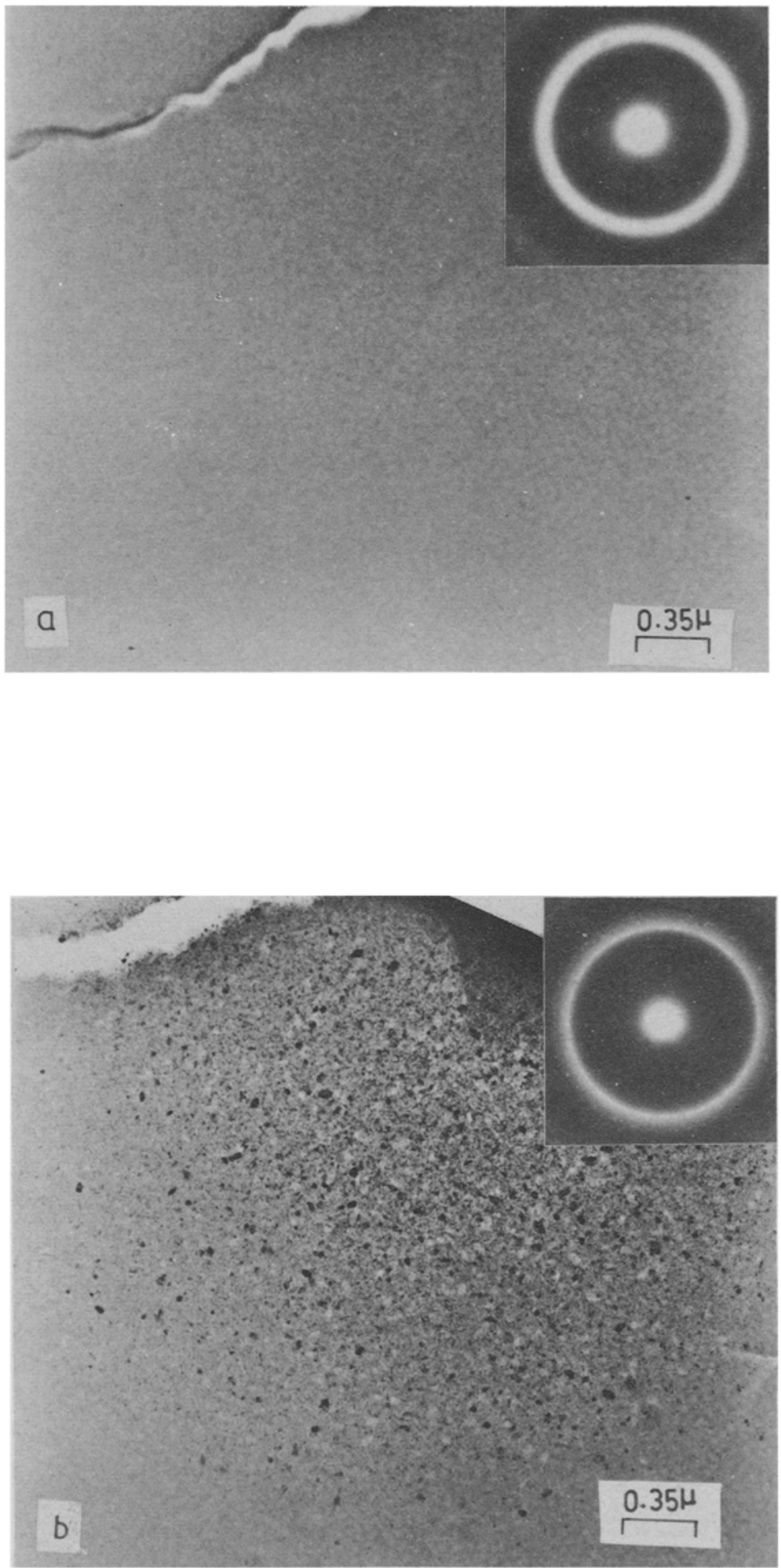

Figure 2. Microstructure and diffraction pattern of HVEM irradiated sample; dose $2 \times 10^{21}$ $\mathrm{e} / \mathrm{cm}^{2}$ a. at room temperature and $\mathbf{b}$. when heated in situ. The crystallisation begins at $653 \mathrm{~K}$. 
valuable information about the micromechanism of the reactions, the crystallisation can be drastically different from that of bulk. We, therefore, irradiated samples in an accelerator and it was observed that crystallisation temperature determined from the bulk measurements such as resistometry were not drastically different from that of their foil measurements.

Comparing our results with those of earlier workers, no definite conclusion about the effect of defects on crystallisation can be drawn. However we can only say that the effects of electron irradiation on $T_{c}$ are closely related to both irradiation conditions and metal-metalloid combination.

\section{Conclusion}

HVEM is being effectively used to study damage in metallic glasses and its influence on the crystallisation temperature using heating-stage of HVEM. However, a HVEM has certain limitations which can be overcome by supplimenting HVEM results with those of irradiation from an electron accelerator. Results on a specific nickel-based alloy $\mathrm{Ni}_{45} \mathrm{Fe}_{5} \mathrm{Co}_{20} \mathrm{Cr}_{10} \mathrm{Mo}_{4} \mathrm{~B}_{16}$ have been discussed and it has been observed that electron irradiation from both HVEM and accelerator has no significant effect on crystallisation temperature.

\section{Acknowledgements}

It is our pleasure to thank $\mathrm{K}$ Varatharajan for his association with this work. Our thanks are due to Dr Ray for providing the metallic glass sample.

\section{References}

Chang B T and Li J C M 1977 Scr. Metall. 11933

Chaung S Y, Tsand P K, Tan G J and Chen H S 1978 Phys. Status Solidi 40 K 181

Chen H S 1976 Phys. Status Solidi 34 K 127

Doi K, Ayan T and Kawamura K 1979 Non-Cryst. Solids 34405

Doi M and Imura T 1978 3rd Int. Conf. on Rapidly Quenched Metals, Brighton (UK) 2372

Doi $M$ and Imura T $1980 \mathrm{~J}$. Mater. Sci. 153701

Doi M, Yoshida M, Nanoyama M, Imura T, Masumato T and Yoshio Y 1976 Mater. Sci. Eng. 23169

Kalumunzer S, Rentzsch S, Schumacher G and Vogl G 1981 Yamada conference on point defects and defect interactions, Kyoto (Japan)

Kalumunzer S and Petri W 1982 Phys. Lett. A87 314

Kiritani M and Yoshiie T 1978 Suppl. Sci. Rep. Research Institute of Tôhoku University, Japan A63

Kiritani M, Yoshiie T and Fujita F E 1977 Proc. V Int. Conf. on High Voltage Electron Microscopy, Kyoto (Japan)

Kiritani M, Yoshiie T and Fujita F E 1978 Proc. II Int. Conf. on Rapidly Quenched Metals, Brighton (UK) 2308

Koster U and Herald U 1981 Glassy metals I (eds) H J Guntherodt and H J Beck (Berlin: Springer-Verlag) 225

Kramer E A, Johnson W L and Cline C 1979 Appl. Phys. Lett. 35815

Lesueur D 1970 Fizika 2 Suppl. 2131

Lesueur D 1975 Radiat. Eff. 24101

Makin M J 1971 Electron microscopy in materials science (ed) U Valdre (New York and London: Academic Press) 388

Moser P, Hautojarvi P, Yli-Kaupilla J and Chamberrod A 1981 4th Int. Conf. on Rapidly Quenched Metals, Sendai (Japan) 
Nandedkar R V and Tyagi A K 1981a Radiat. Eff. Lett. 5891

Nandedkar R V and Tyagi A K 1981 b Scr. Metall. 151175

Nandedkar R V, Tyagi A K and Varatharajan K 1981 4th Int. Conf. on Rapidly Quenched Metals, Sendai (Japan)

Norris D I R 1972a Radiat. Eff. 141

Norris D I R 1972b Radiat. Eff. 151

Richtin M, Van der Sande J and Baldo P M 1978 Scr. Metall. 12639

Schumacher G, Klaumunzer Rentzsch S and Vogl G 1980 Z. Phys. B40 19

Von Heimendale 1981 Scr. Metall. 151145 